\title{
Cardiac Computed Tomography for the detection of cardiac amyloidosis
}

Short title: Cardiac CT for detection of cardiac amyloidosis

Stefania Rosmini, MD, $\mathrm{PhD}^{\mathrm{ab} *}$, Thomas A Treibel, MBBS ${ }^{\mathrm{b}}$, Steve Bandula, MA MB

BChir $^{\mathrm{c}}$, Tyler Stroud ${ }^{\mathrm{d}}$, Marianna Fontana, MD ${ }^{\mathrm{a}}$, Philip N Hawkins, FMedSci ${ }^{\text {a }}$, James C

Moon, $\mathrm{MD}^{\mathrm{b}}$.

a National Amyloidosis Centre, University College London, Royal Free Hospital, London, UK

${ }^{\mathrm{b}}$ Barts Heart Centre, West Smithfield, London, UK

${ }^{c}$ Centre for Medical Imaging, University College London, London, UK

${ }^{\mathrm{d}}$ Toshiba Medical Visualization Systems Europe Ltd, Edinburgh, UK

* Joint first authors

Corresponding author:

Professor James Moon

Barts Heart Centre

St. Bartholomew's Hospital

West Smithfield, London EC1A 7BE

T: +442034563081

F: +442034563086

E:j.moon@ucl.ac.uk

Word count: 300

\section{Conflicts of interest}

None 
A 67-year-old Caucasian man presented with atrial flutter on routine 12-lead electrocardiography (ECG). Following appropriate anticoagulation, he successfully underwent atrial flutter ablation. Echocardiography during the admission showed concentric left ventricular (LV) hypertrophy with severely impaired LV systolic function, particularly longitudinal (Figure 1A, video 1), and biatrial dilatation. Due to episodes of non-sustained ventricular tachycardia, he was fitted with an implantable cardioverter-defibrillator (ICD). The ECG QRS complexes on peripheral leads were small compared to the structural wall thickening on echocardiography (Figure 1B) raising the suspicion of cardiac amyloidosis. Preliminary evaluation excluded a plasma cell dyscrasia (negative serum free light chains changes and urinary Bence-Jones protein). Serum amyloid P component (SAP) scintigraphy was negative for visceral organ uptake and bone tracer scintigraphy (Technetium-DPD) was positive with Perugini Grade 2-3 uptake (Figure 1C), consistent with a diagnosis of cardiac transthyretin amyloidosis (ATTR). Cardiac magnetic resonance (CMR) was contra-indicated due to the non-conditional ICD (Figure 1D). After giving fully informed written consent, cardiac computed tomography (CCT) was performed as part of a research study, using a three step protocol (Figure 1E) as previously described ${ }^{1}$, allowing calculation of the extracellular volume fraction (ECV) from pre- and 5-minutes post-contrast images. The septal ECV, calculated from a co-registered, segmented myocardial mask displaying pixel-by-pixel ECV values, was in the amyloid spectrum elevated at $63 \%$ (Figure $1 \mathrm{~F}$ ), Gene sequencing revealed a heterozygous V122I mutation, confirming the diagnosis of familial amyloid cardiomyopathy (ATTR).

\section{Discussion}

Quantification of the myocardial ECV using two-phase cardiac CT can discriminate between cardiac amyloidosis and other disease with myocardial hypertrophy. The ECV by CMR and CT strongly correlated, making CCT a valid alternative to $\mathrm{CMR}^{1}$, which itself is known to 
have good sensitivity and specificity for the diagnosis, prognostication and monitoring of cardiac amyloidosis ${ }^{2}$. Here, CCT added value as there was a MR non-conditional ICD in-situ.

\section{Fundings:}

S.R. is supported by Borse di studio SIC e MSD Italia-Merck Sharp \& Dohme. T.A.T is supported by Doctoral Research Fellowship 280 from the NIHR, UK (NIHR-DRF- 2013-06102). 


\section{References}

1. Treibel TA, Bandula S, Fontana M, et al. Extracellular volume quantification by dynamic equilibrium cardiac computed tomography in cardiac amyloidosis. Journal of cardiovascular computed tomography. 2015;9:585-592.

2. Fontana M, Pica S, Reant P, et al. Prognostic Value of Late Gadolinium Enhancement Cardiovascular Magnetic Resonance in Cardiac Amyloidosis. Circulation. 2015;132:15701579.
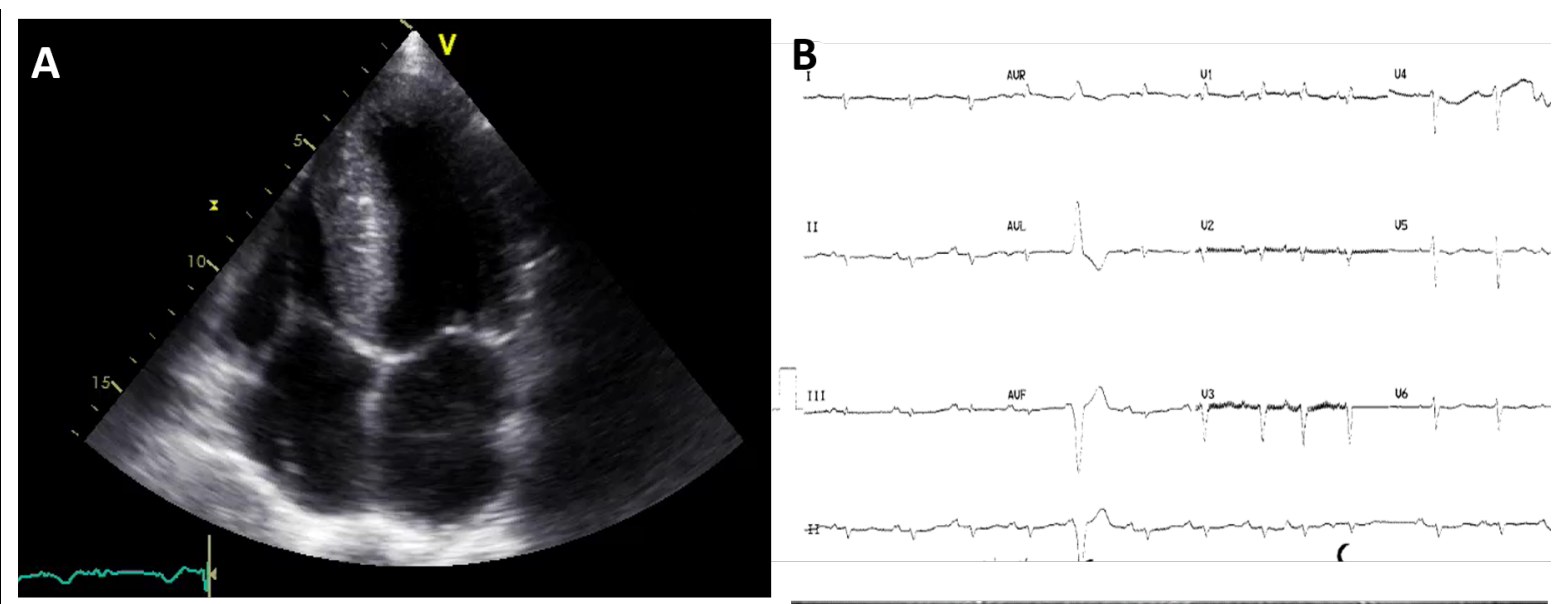

C

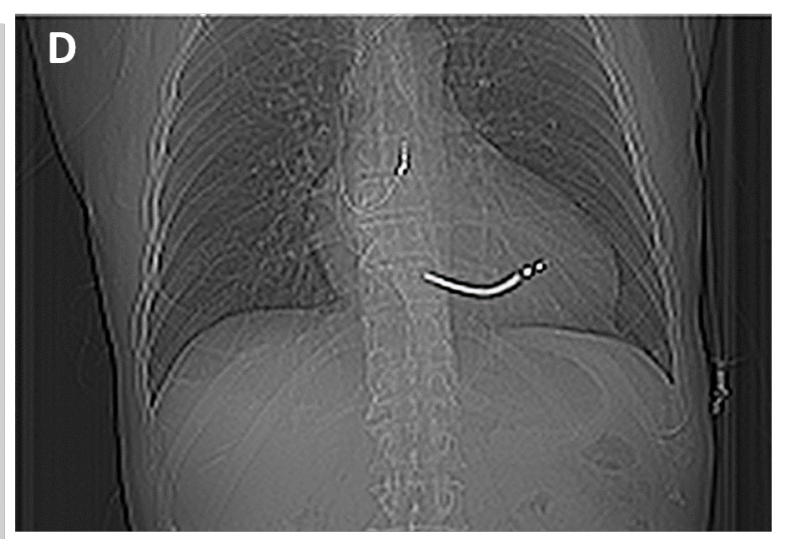

\section{E I. Acquisition} Pre-contrast Angiographic Post-contrast

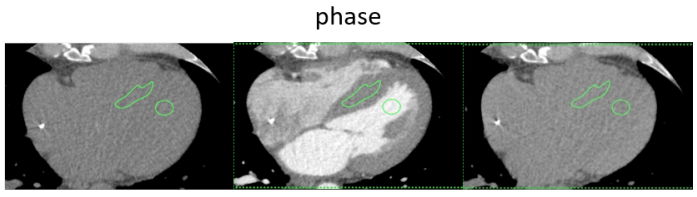

II. Co-registration and Segmentation (myocardial mask)

III. ECV volume fused with angiographic phase (MPR)
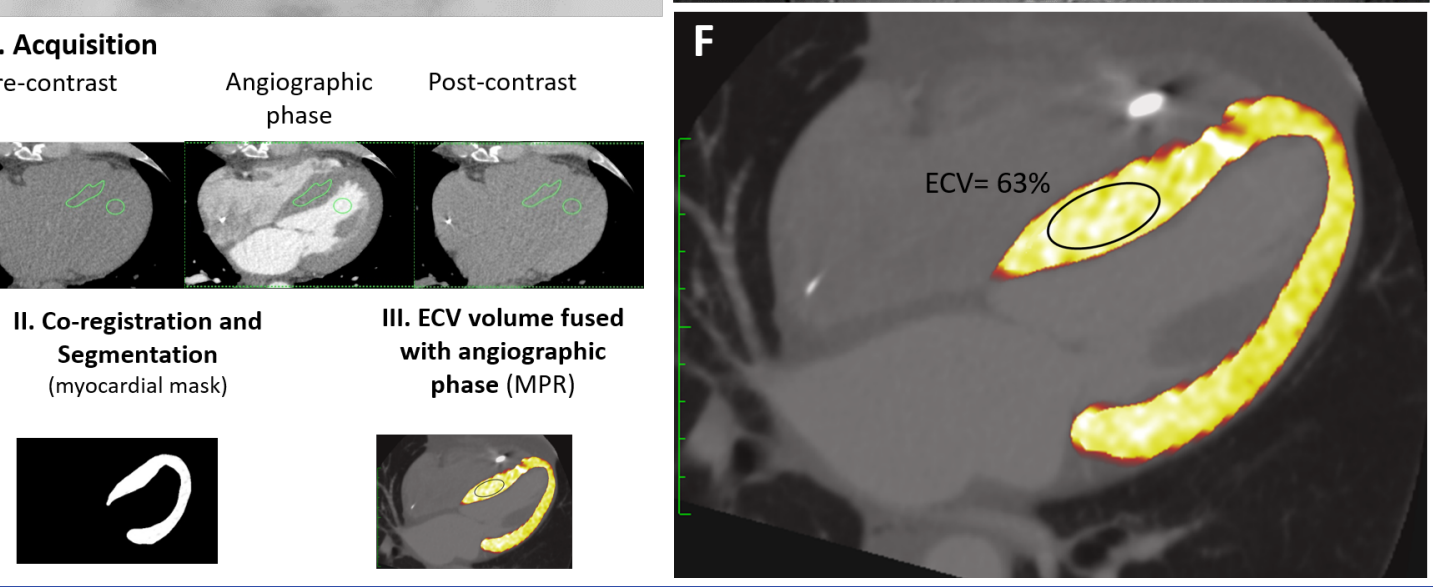